\title{
A PAIR OF DUAL INTEGRAL EQUATIONS IN- VOLVING BESSEL FUNCTIONS OF THE FIRST AND THE SECOND KIND
}

\author{
by R. P. SRIVASTAV
}

(Received 20th January 1964)

\section{Introduction}

In this paper we give a method for the solution of the dual integral equations

$$
\begin{gathered}
\int_{0}^{\infty} \xi \psi(\xi) \frac{J_{v}(\xi \rho) Y_{v}(\xi)-Y_{v}(\xi \rho) J_{v}(\xi)}{J_{v}^{2}(\xi)+Y_{v}^{2}(\xi)} d \xi=f_{1}(\rho), \quad 1<\rho<a \\
\int_{0}^{\infty} \xi^{2 \alpha+1} \psi(\xi) \frac{J_{v}(\xi \rho) Y_{v}(\xi)-Y_{v}(\xi \rho) J_{v}(\xi) d \xi}{J_{v}^{2}(\xi)+Y_{v}^{2}(\xi)}=-f_{2}(\rho), \quad \rho>a
\end{gathered}
$$

where $J_{v}$ and $Y_{v}$ are Bessel functions of the first and second kind, $-\frac{1}{2} \leqq \alpha \leqq \frac{1}{2}$, $f_{1}(\rho)$ and $f_{2}(\rho)$ are known functions and $\psi(\xi)$ is to be determined. Such equations arise in the discussion of boundary value problems for half-spaces containing a cylindrical cavity. For example, let us take the problem of finding a potential function $\phi(\rho, \theta, z)$ which satisfies Laplace's equation for

$$
1<\rho<\infty, 0 \leqq \theta \leqq 2 \pi, z>0
$$

subject to the usual regularity conditions and the following boundary conditions :

$$
\begin{aligned}
& \phi(1, \theta, z)=0, \quad 0 \leqq \theta \leqq 2 \pi, \quad z>0 \\
& \phi(\rho, \theta, 0)=0, \quad 0 \leqq \theta \leqq 2 \pi, \quad 1<\rho<a \\
& \frac{\partial}{\partial z} \phi(\rho, \theta, z)=\left(f_{m}(\rho) \cos m \theta+g_{m}(\rho) \sin m \theta\right) \\
& 0 \leqq \theta \leqq 2 \pi, \quad \rho>a \text { and } z=0 \text {. }
\end{aligned}
$$

A suitable representation for such a function is

$$
\begin{aligned}
& \phi(\rho, \theta, \mathrm{z}) \\
& =\int_{0}^{\infty} \tilde{\zeta} e^{-\xi^{\xi} z} \frac{\left(\psi_{c}(\xi) \cos m \theta+\psi_{s}(\xi) \sin m \theta\right)\left(J_{m}(\zeta \rho) Y_{m}(\xi)-Y_{m}(\xi \rho) J_{m}(\xi)\right)}{J_{m}^{2}(\xi)+Y_{m}^{2}(\xi)} d \xi .
\end{aligned}
$$

A function expressible in the above form satisfies the condition (1.3) for all $\psi$ 's for which the integral (1.6) is convergent. The conditions (1.4) and (1.5) are fulfilled if $\psi_{c}$ and $\psi_{s}$ are found such that

$$
\int_{0}^{\infty} \xi \psi_{c}(\xi) \frac{J_{m}(\xi \rho)}{J_{m}^{2}(\xi)+Y_{m}^{2}(\xi)} d \xi=0, \quad 1<\rho<a,
$$




$$
\int_{0}^{\infty} \xi^{2} \psi_{c}(\xi) \frac{J_{m}(\xi \rho) Y_{m}(\xi)-Y_{m}(\xi \rho) J_{m}(\xi)}{J_{m}^{2}(\xi)+Y_{m}^{2}(\xi)} d \xi=-f_{m}(\rho), \quad \rho>a
$$

and a similar pair of equations holds for $\psi_{s}$.

Weber (1) wrote down, by inspection, the solution of the pair of integral equations

$$
\begin{aligned}
& \int_{0}^{\infty} \xi^{\alpha} \psi(\xi) J_{v}(\xi \rho) d \xi=f(\rho), \quad 0<\rho<1, \\
& \int_{0}^{\infty} \psi(\xi) J_{v}(\xi \rho) d \xi=g(\rho), \quad 1<\rho<\infty .
\end{aligned}
$$

for

$$
v=0, \alpha=-1, f(\rho)=1, g(\rho)=0 .
$$

This could be regarded as the starting point of the theory of dual integral equations. The first direct solution for the same parameters but with $f$ a non-constant function of $\rho$ appears to be due to Beltrami (2).

The problem for general values of the parameters but $g(\rho)=0$ was considered by Titchmarsh (3), Busbridge (4) and Gordon (5). The analysis in these solutions is rather complicated and does not easily lend itself to adaptation to cover more general cases. An elementary method was suggested by Sneddon (6) for certain special cases arising from the consideration of boundary value problems in mathematical physics. An elegant generalisation of Sneddon's method was used by Copson (7) to solve the problem in the general case.

The solution of the pair of equations (1.9) and (1.10) for $-1 \leqq \alpha \leqq 1$ was considered by Noble (8) who reduced the problem to one of solving an integral equation of Abel-Schlömilch type. Tranter (9) had earlier derived a solution for the case $\alpha= \pm 1$ but his method is no simpler than Noble's. The solution derived by Williams (10) by a formal application of the theory of Mellin transforms is apparently simpler but, as pointed out by Erdélyi and Sneddon (11), it is so because much labour has already been absorbed in calculating the transforms. The last-mentioned authors have tried to bring out, by introducing fractional integration operators, the relationship which exists between various solutions. Recently Lowengrub and Sneddon (12) have given an easy method for dealing with the equations (1.9) and (1.10) where $f(\rho)=0$ and their paper may be treated as a complement to that of Copson (7).

The most notable contribution to the theory in the recent past has however been made by Williams (13) who associated the solution of the dual integral equations with the factorisation of linear operators. The analogous problem of determining the coefficients in dual series relations.

$$
\begin{array}{r}
\sum_{n=1}^{\infty} \lambda_{n}^{-p} a_{n} J_{v}\left(\lambda_{n} \rho\right)=f(\rho), \quad 0<\rho<1, \\
\sum_{n=1}^{\infty} a_{n} J_{v}\left(\lambda_{n} \rho\right)=0, \quad 1<\rho<a, .
\end{array}
$$


where $-1 \leqq p \leqq 1, v>-1$ and $\lambda_{n}$ are positive zeros of $J_{v}(a \lambda)$, was first discussed by Cooke and Tranter (14), who showed that the dual series equations may be replaced by an infinite set of linear algebraic equations. Recently Sneddon and Srivastav (15) reduced the above problem to one of solving a Fredholm integral equation of the second kind. In elementary solutions of dual integral equations, a form for $\psi(\xi)$ was chosen which satisfied one of the equations identically. The key idea in the paper by Sneddon and Srivastav is that of assuming an integral representation for the infinite series in (1.12) and thus automatically deriving a suitable representation for the coefficients. In this paper we have combined this idea with the idea of factorisation introduced by Williams (without explicitly stating it in the text) and have thus succeeded in reducing the pair of equations (1.1) and (1.2) to a single linear integral equation of Fredholm type of the second kind. The special case $v=0, \alpha=\frac{1}{2}$ and $f_{1}(\rho) \equiv 0$ has been discussed in an earlier paper and we shall exclude these values of the parameters.

To facilitate the presentation we split the problem of determining $\psi(\xi)$, which satisfies the equations (1.1) and (1.2), into two problems:

Problem (a). Determine $\psi(\xi)$ which satisfies the equations

$$
\begin{gathered}
\int_{0}^{\infty} \xi \psi(\xi) \frac{J_{v}(\xi \rho) Y_{v}(\xi)-Y_{v}(\xi \rho) J_{v}(\xi)}{J_{v}^{2}(\xi)+Y_{v}^{2}(\xi)} d \xi=0, \quad 1<\rho<a \\
\int_{0}^{\infty} \xi^{2 \alpha+1} \psi(\xi) \frac{J_{v}(\xi \rho) Y_{v}(\xi)-Y_{v}(\xi \rho) J_{v}(\xi)}{J_{v}^{2}(\xi)+Y_{v}^{2}(\xi)} d \xi=-f_{2}(\rho), \quad \rho>a \ldots .
\end{gathered}
$$

Problem (b). Determine $\psi(\xi)$ which satisfies the equations

$$
\begin{gathered}
\int_{0}^{\infty} \xi \psi(\xi) \frac{J_{v}(\xi \rho) Y_{v}(\xi)-Y_{v}(\xi \rho) J_{v}(\xi)}{J_{v}^{2}(\xi)+Y_{v}^{2}(\xi)} d \xi=f_{1}(\rho), \quad 1<\rho<a, \\
\int_{0}^{\infty} \xi^{2 \alpha+1} \psi(\xi) \frac{J_{v}(\xi \rho) Y_{v}(\xi)-Y_{v}(\xi \rho) J_{v}(\xi)}{J_{v}^{2}(\xi)+Y_{v}^{2}(\xi)} d \xi=0, \rho>a .
\end{gathered}
$$

The solution to the general problem can obviously be obtained by adding the two solutions. In view of the heavy calculations involved in our work here, it appears advisable to recast some of the well-known properties of Bessel functions in a form more suitable for our purpose. This is done in Section 2. Sections $\mathbf{3}$ and $\mathbf{4}$ are devoted to the solutions of problems (a) and (b) respectively (in the sense of reduction to a Fredholm integral equation of the second kind). We outline an approach in Section 5 for the solution of the dual equations when $\xi^{2 \alpha+1}$ in the equation (1.2) is replaced by $\xi^{2 \alpha+1}(1+H(\xi))$, where $H(\xi)$ is a known function. Such a pair of equations is the counterpart of the equations discussed by Tranter (17) and (17a), Cooke (18) and Noble (19).

The analysis throughout this paper is formal and no attempt is made to justify the change of order of integrations. The conditions, whenever written explicitly, are not in the sharpest form and are usually dictated by the consideration of convergence of integrals. 
2. Let

$$
\chi_{\mu, v}(\rho, \xi) \equiv J_{\mu}(\rho \xi) Y_{v}(\xi)-Y_{\mu}(\rho \xi) J_{v}(\xi)
$$

It may be easily verified that

$$
\chi_{\mu, v}(\rho, \xi)=-\frac{1}{2 i}\left(\frac{H_{\mu}^{(1)}(\rho \xi)}{H_{v}^{(1)}(\xi)}-\frac{H_{\mu}^{(2)}(\rho \xi)}{H_{v}^{(2)}(\xi)}\right) H_{v}^{(1)}(\zeta) H_{v}^{(2)}(\xi)
$$

where $H^{(1)}$ and $H^{(2)}$ are Bessel functions of the third kind defined by the relations [(20), p. 73]

$$
\begin{aligned}
& H_{v}^{(1)}(z)=J_{v}(z)+i Y_{v}(z), \\
& H_{v}^{(2)}(z)=J_{v}(z)-i Y_{v}(z) .
\end{aligned}
$$

From the formulæ (32), p. 25 and (38), p. 104 of (21), it may be deduced that

$$
\int_{t}^{\infty} \rho^{1-v} \chi_{\mu, v}(\rho, \xi)\left(\rho^{2}-t^{2}\right)^{\alpha} d \rho=2^{x} \Gamma(\alpha+1) t^{1+\alpha-v} \xi^{-\alpha-1} \chi_{\mu-\alpha-1, v}(t, \xi),
$$

a result which is valid for $R(\mu)>-1$ and $R(\mu-2 \alpha)>\frac{1}{2}$.

We call $\bar{g}(\xi)$ the Weber transform of first kind of order $v$ of $g(\rho)$ if

$$
W_{v}(g) \equiv \bar{g}(\xi)=\int_{1}^{\infty} \rho g(\rho) \chi_{v, v}(\rho, \xi) d \rho, \quad 0<\xi<\infty
$$

It easily follows from (22), p. 75 that

$$
W_{v}^{-1}(\bar{g}) \equiv g(\rho)=\int_{0}^{\infty} \frac{\xi \bar{g}(\xi) \chi_{v, v}(\rho, \xi) d \xi}{H_{v}^{(1)}(\xi) H_{v}^{(2)}(\xi)} .
$$

Combining the relations (2.5) and (2.7) we find that

$$
\int_{0}^{\infty} \xi^{-\alpha} \frac{\chi_{v-\alpha-1, v}(t, \xi) \chi_{v, v}(\rho, \zeta)}{H_{v}^{(1)}(\xi) H_{v}^{(2)}(\xi)} d \xi=\left\{\begin{array}{l}
0, \quad 1<\rho<t, \\
\frac{2^{-\alpha} t^{\nu-\alpha-1}\left(\rho^{2}-t^{2}\right)^{\alpha}}{\Gamma(\alpha+1) \rho^{v}}, \rho>t .
\end{array}\right.
$$

There is a striking resemblance between the integral (2.8) and the following special case of the Weber-Schafheitlin integral [(21), (8), p. 48]: For $v>\mu>-1$,

$$
\int_{0}^{\infty} x^{\mu-v+1} J_{\mu}(a x) J_{v}(x y) d x=\left\{\begin{array}{l}
0, \quad 0<y<a \\
\frac{2^{\mu-v+1} a^{\mu}\left(y^{2}-a^{2}\right)^{-\mu-1}}{\Gamma(v-\mu) y^{v}}, a<y<\infty .
\end{array}\right.
$$

We can show easily that

$$
\begin{aligned}
& \int_{0}^{\infty} \xi^{\alpha+1} \frac{\chi_{\eta, v}(\rho, \xi) \chi_{\mu, v}(t, \xi) d \xi}{H_{v}^{(1)}(\xi) H_{v}^{(2)}(\xi)} \\
& \quad=R\left(\int_{0}^{\infty} \xi^{\alpha+1} \frac{H_{\eta}^{(1)}(\rho \xi) H_{\mu}^{(1)}(t \xi)}{H_{v}^{(1)}(\xi)} J_{v}(\xi) d \xi\right)+\int_{0}^{\infty} \xi^{\alpha+1} J_{\eta}(\rho \xi) J_{\mu}(t \xi) d \xi .
\end{aligned}
$$

The second integral in the equation (2.10) is the Weber-Schafheitlin integral 
whose evaluation has been discussed by Watson [(20), p. 398]. By considering

$$
\int_{\Gamma} z^{a+1} J_{v}(z) \frac{H_{\eta}^{(1)}(\rho z) H_{\mu}^{(1)}(t z)}{H_{v}^{(1)}(z)} d z
$$

where $\Gamma$ consists of the positive real axis, the arc of the quadrant and the positive imaginary axis, we can show that

$$
\begin{aligned}
& R\left(\int_{0}^{\infty} \xi^{\alpha+1}\right.\left.\frac{J_{v}(\xi) H_{\eta}^{(1)}(\rho \xi) H_{\mu}^{(1)}(t \xi)}{H_{v}^{(1)}(\xi)} d \xi\right) \\
& \quad=\frac{2}{\pi} \cos (2 v+\alpha+1-\eta-\mu) \frac{\pi}{2} \int_{0}^{\infty} y^{\alpha+1} \frac{I_{v}(y)}{K_{v}(y)} K_{\eta}(\rho y) K_{\mu}(t y) d y .
\end{aligned}
$$

Thus when $(2 v+\alpha-\eta-\mu)$ is equal to an even integer or zero,

$$
H_{v, \eta, \mu, a}(\rho, t) \equiv \int_{0}^{\infty} \xi^{\alpha+1} \frac{\chi_{\eta, v}(\rho, \xi) \chi_{\mu, v}(t, \xi)}{H_{v}^{(1)}(\xi) H_{v}^{(2)}(\xi)} d \xi
$$

reduces to the Weber-Schafheitlin integral.

\section{Solution of the problem (a). Case $-\frac{1}{2} \leqq \alpha<0$.}

Let us now consider the solution of the equations (1.13) and (1.14) when $-\frac{1}{2} \leqq x<0$. Set

$$
W_{v}^{-1}(\psi(\xi))=\rho^{-v-1} \frac{\partial}{\partial \rho} \int_{a}^{\rho} g(t)\left(\rho^{2}-t^{2}\right)^{x} d t, \quad \rho>a,
$$

where $g(t)$ is as yet an unspecified function. From the relation (2.5) it follows that

$$
\psi(\xi)=2^{\alpha} \Gamma(\alpha+1) \xi^{-\alpha} \int_{a}^{\infty} g(t) t^{\alpha-v} \chi_{v-\alpha, v}(t, \xi) d t .
$$

The form (3.2) for $\psi(\xi)$ satisfies the equation (1.13) identically. Replacing $\psi(\xi)$ in the equation (1.14) by the value given by (3.2) we get, on interchanging the order of integrations, the following equation to determine $g(t)$ :

$$
2^{\alpha} \Gamma(\alpha+1) \int_{a}^{\infty} g(t) t^{\alpha-v} H_{v, v, v-\alpha, \alpha}(\rho, t) d t=-f_{2}(\rho), \quad \rho>a .
$$

(3.3) is a Fredholm integral equation of the first kind. On combining the relations (2.9), (2.10) and (2.12) we find that

$H_{v, v, v-\alpha, \alpha}(\rho, t)$

$=\frac{2^{\alpha+1}}{\Gamma(-\alpha)} \frac{\rho^{v}\left(t^{2}-\rho^{2}\right)^{-\alpha-1}}{t^{v-\alpha}} H(t-\rho)+\frac{2}{\pi} \sin \pi \alpha \int_{0}^{\infty} y^{\alpha+1} \frac{I_{v}(y)}{K_{v}(y)} K_{v-\alpha}(t y) K_{v}(\rho y) d y$,

where $H(x)$ is Heaviside's unit function. Thus the equation (3.3) is equivalent E.M.S. - L 
to the equation

$$
\begin{aligned}
\frac{2^{-\alpha} \Gamma(-\alpha)}{\pi \operatorname{cosec} \pi \alpha} \int_{a}^{\infty} g(t) t^{z-v}\left(\int_{0}^{\infty} y^{\alpha+1} \frac{I_{v}(y)}{K_{v}(y)} K_{v-\alpha}(t y) \rho^{-v} K_{v}(\rho y) d y\right) d t \\
+\int_{\rho}^{\infty} g(t) t^{2(\alpha-v)}\left(t^{2}-\rho^{2}\right)^{-\alpha-1} d t=\frac{-f_{2}(\rho) \rho^{-v} \Gamma(-\alpha)}{2^{2 \alpha+1} \Gamma(\alpha+1)} .
\end{aligned}
$$

By a procedure similar to that of solving integral equations of Abel-type, we can show that

$$
\begin{array}{r}
g(t) t^{\alpha-\nu}+\frac{2 t}{\pi} \sin \pi \alpha \int_{a}^{\infty} g(u) u^{\alpha-\nu}\left(\int_{0}^{\infty} \frac{y I_{v}(y)}{K_{v}(y)} K_{v-\alpha}(u y) K_{\alpha-v}(t y) d y\right) d u \\
=\frac{2^{-2 \alpha} t^{\nu-\alpha}}{\{\Gamma(1+\alpha)\}^{2}} \frac{d}{d t} \int_{t}^{\infty} \rho^{1-v} f_{2}(\rho)\left(\rho^{2}-t^{2}\right)^{\alpha} d \rho, \ldots
\end{array}
$$

where the simplification is to be effected by the use of the following integral [(21), (13), p. 129]: For $\boldsymbol{R}(\mu)>-1$ and $\boldsymbol{R}(y)>0$

$$
\int_{a}^{\infty} x^{1-v}\left(x^{2}-a^{2}\right)^{\mu} K_{v}(x y) d x=2^{\mu} a^{\mu-v+1} y^{-\mu-1} \Gamma(\mu+1) K_{\mu-v+1}(a y) .
$$

For $v=\frac{1}{2}$, it is possible to find a closed form solution for the equations (1.1) and (1.2). It is however not clear how the solution of the above Fredholm integral equation is related to such a solution.

Case $0<\alpha \leqq \frac{1}{2}$.

We make a further assumption that $v>0$. For $v=0$, the case of physical significance appears to be the one corresponding to $\alpha=\frac{1}{2}$. The solution for these values of the parameters has already been discussed in (16). For $v>0$ and $0<\alpha \leqq \frac{1}{2}$, we suppose that

$$
W_{v}^{-1}(\psi(\xi))=\rho^{-v} \int_{a}^{\rho} g(t)\left(\rho^{2}-t^{2}\right)^{\alpha-1} d t, \quad \rho>a .
$$

This is equivalent to the assumption that

$$
\psi(\xi)=2^{\alpha-1} \Gamma(\alpha) \xi^{-\alpha} \int_{a}^{\infty} t^{\alpha-v} g(t) \chi_{v-\alpha, v}(t, \xi) d t .
$$

Multiplying both sides of the equation (1.14) by $\rho^{1-v}$ and integrating from $\rho$ to $\infty$, we obtain the equation

$$
\int_{0}^{\infty} \xi^{2 \alpha} \frac{\psi(\xi) \chi_{v-1, v}(\rho, \xi)}{H_{v}^{(1)}(\xi) H_{v}^{(2)}(\xi)} d \xi=-\rho^{v-1} \int_{\rho}^{\infty} f_{2}(u) u^{1-v} d u, \quad \rho>a .
$$

If we substitute the value of $\psi(\xi)$ from the equation (3.9) in the equation (3.10), we obtain on interchanging the order of integration, the equation

$$
\begin{aligned}
2^{\alpha-1} \Gamma(\alpha) \int_{a}^{\infty} t^{\alpha-v} g(t) H_{v, v-1, v-\alpha, \alpha-1}(\rho, t) d t & \\
& =-\rho^{v-1} \int_{\rho}^{\infty} f_{2}(u) u^{1-v} d u, \quad \rho>a .
\end{aligned}
$$


From the relations (2.9), (2.10) and (2.12) it is easily deduced that

$$
\begin{aligned}
& H_{v, v-1, v-\alpha, \alpha-1}(\rho, t) \\
& \quad=\frac{2^{\alpha} \rho^{v-1} t^{\alpha-v}\left(t^{2}-\rho^{2}\right)^{-\alpha} H(t-\rho)}{\Gamma(1-\alpha)}-\frac{2}{\pi} \sin \pi \alpha \int_{0}^{\infty} y^{\alpha} \frac{K_{v-1}(\rho y) K_{v-\alpha}(y t) I_{v}(y)}{K_{v}(y)} d y .
\end{aligned}
$$

This reduces the equation (3.11) to the equation

$$
\begin{gathered}
2^{-\alpha} \Gamma(1-\alpha) \cdot \frac{2}{\pi} \sin \pi \alpha \int_{a}^{\infty} g(u) u^{\alpha-v}\left(\int_{0}^{\infty} \frac{y^{\alpha} K_{v-1}(\rho y) \rho^{1-v} K_{v-\alpha}(u y) I_{v}(y)}{K_{v}(y)} d y\right) d u \\
-\int_{\rho}^{\infty} t^{2 \alpha-2 v} g(t)\left(t^{2}-\rho^{2}\right)^{-\alpha} d t=\frac{2^{1-2 \alpha} \Gamma(1-\alpha)}{\Gamma(\alpha)} \int_{\rho}^{\infty} f(u) u^{1-v} d u, \quad \rho>a \ldots .(3.13)
\end{gathered}
$$

The above equation may be easily shown to be equivalent to the equation

$$
\begin{aligned}
g(t) t^{\alpha-\nu}+\frac{2 t}{\pi} \sin \pi \alpha \int_{a}^{\infty} & g(u) u^{\alpha-v}\left(\int_{0}^{\infty} y \frac{I_{v}(y)}{K_{v}(y)} K_{v-\alpha}(u y) K_{\alpha-v}(t y) d y\right) d u \\
& =\frac{2^{1-2 \alpha} \alpha t^{\nu-\alpha}}{\{\Gamma(H-\alpha)\}^{2}} \cdot \frac{d}{d t} \int_{t}^{\infty} f_{2}(u) u^{1-v}\left(u^{2}-t^{2}\right)^{\alpha} d u .
\end{aligned}
$$

Thus the integral equation obtained is of the same type as (3.6), though the derivation is slightly different.

4. Solution of the problem (b). Case $-\frac{1}{2} \leqq \alpha<0$.

The procedure for solving this problem is essentially the same as that for problem (a). Let us assume that for $\rho>a, W_{v}^{-1}(\psi(\xi))$ is given by the equation (3.1). Then

$$
\psi(\xi)=2^{\alpha} \Gamma(\alpha+1) \xi^{-\alpha} \int_{a}^{\infty} g(t) t^{\alpha-v} \chi_{v-\alpha, v}(t, \xi) d t+\int_{1}^{a} \rho f_{1}(\rho) \chi_{v, v}(\rho, \xi) d \rho .
$$

If we substitute the above value in (1.16) and interchange the order of integrations, we obtain the equation

$$
2^{\alpha} \Gamma(\alpha+1) \int_{a}^{\infty} g(t) t^{\alpha-v} H_{v, v, v-\alpha, \alpha}(\rho, t) d t=-\int_{1}^{a} v f_{1}(v) H_{v, v, v, 2 \alpha-1}(v, \rho) d v, \rho>a,
$$

which is of the same form as the equation (3.3). Hence we can reduce it to the Fredholm Integral equation of the second kind

$$
\begin{gathered}
g(t) t^{\alpha-v}+\frac{2 t}{\pi} \sin \pi \alpha \int_{a}^{\infty} g(u) u^{\alpha-v}\left(\int_{0}^{\infty} y \frac{I_{v}(y)}{K_{v}(y)} K_{v-\alpha}(u y) K_{v-\alpha}(t y) d y\right) d u \\
=-\frac{2^{-\alpha} t}{\Gamma(1+\alpha)} \int_{1}^{a} v f_{1}(v) H_{v, v, v-\alpha, \alpha-1}(v, t) d v, \quad a<t<\infty,
\end{gathered}
$$

by using the result (2.5). The case $0<\alpha \leqq \frac{1}{2}$ can also be treated in the same manner and results in the same integral equation. The equation (4.3) is much 
more difficult to handle than the equations (3.6) or (3.14). Not only is the kernel of the integral equation an integral but here the free function involves a rather complicated double integral. Nevertheless, the problem is solved, at least in principle.

5. Let us now consider the solution of the pair of equations

$$
\begin{array}{r}
\int_{0}^{\infty} \frac{\xi \psi(\xi) \chi_{v, v}(\rho, \xi)}{J_{v}^{2}(\xi)+Y_{v}^{2}(\xi)} d \xi=0, \quad 1<\rho<a, \ldots \ldots \ldots . \\
\int_{0}^{\infty} \xi^{2 x+1} \frac{(1+H(\xi)) \psi(\xi) \chi_{v}, v(\rho, \xi)}{J_{v}^{2}(\xi)+Y_{v}^{2}(\xi)} d \xi=-f_{2}(\rho), \quad \rho>a,
\end{array}
$$

where $-\frac{1}{2} \leqq \alpha \leqq \frac{1}{2}, H(\xi)$ is a known function and $\psi(\xi)$ is to be determined. We rewrite the equation (5.2) in the form

$$
\int_{0}^{\infty} \xi^{2 \alpha+1} \frac{\psi(\xi) \chi_{v, v}(\rho, \xi)}{J_{v}^{2}(\xi)+Y_{v}^{2}(\xi)} d \xi=-F_{2}(\rho), \quad \rho>a,
$$

where

$$
F_{2}(\rho)=f_{2}(\rho)+\int_{0}^{\infty} \xi^{2 \alpha+1} \frac{H(\xi) \psi(\xi) \chi_{v, v}(\rho, \xi)}{J_{v}^{2}(\xi)+Y_{v}^{2}(\xi)} d \xi
$$

For $-\frac{1}{2} \leqq \alpha<0$

$$
\psi(\xi)=2^{\alpha} \Gamma(\alpha+1) \xi^{-\alpha} \int_{a}^{\infty} g(t) t^{\alpha-v} \chi_{v-\alpha, v}(t, \xi) d t
$$

and for $0<\alpha \leqq \frac{1}{2}$

$$
\psi(\xi)=2^{\alpha-1} \Gamma(\alpha) \xi^{-\alpha} \int_{a}^{\infty} g(t) t^{\alpha-v} \chi_{v-\alpha, v}(t, \zeta) d t
$$

where $g(t)$ satisfies the Fredholm integral equation of the second kind

$$
\begin{gathered}
g(t) t^{\alpha-v}+\frac{2 t}{\pi} \sin \pi \alpha \int_{a}^{\infty} g(u) u^{\alpha-v}\left(\int_{0}^{\infty} y \frac{I_{v}(y)}{K_{v}(y)} K_{v-\alpha}(u y) K_{\alpha-v}(t y) d y\right) d u \\
=\frac{2^{-2 \alpha} t^{v-\alpha}}{\{\Gamma(1+\alpha)\}^{2}} \frac{d}{d t} \int_{t}^{\infty} \rho^{1-v} F_{2}(\rho)\left(\rho^{2}-t^{2}\right)^{\alpha} d \rho, \quad a<t<\infty .
\end{gathered}
$$

However $F_{2}(\rho)$ involves $\psi(\xi)$ and therefore $g(t)$. The equation (5.7) can be shown to be equivalent to the equation

$$
\begin{aligned}
g(t) t^{\alpha-v}+\frac{2 t}{\pi} \sin \pi \alpha \int_{a}^{\infty} g(u) u^{\alpha-v}\left(L_{1}(u, t)+L_{2}(u, t)\right) d u \\
=\frac{2^{-2 \alpha} t^{y-\alpha}}{\{\Gamma(1+\alpha)\}^{2}} \frac{d}{d t} \int_{t}^{\infty} \rho^{1-v} f_{2}(\rho)\left(\rho^{2}-t^{2}\right)^{\alpha} d \rho, \quad a<t<\infty
\end{aligned}
$$


where

$$
L_{1}(u, t)=\int_{0}^{\infty} y \frac{I_{v}(y)}{K_{v}(y)} K_{v-\alpha}(u y) K_{v-\alpha}(t y) d y, \quad a<u, t<\infty
$$

and

$$
L_{2}(u, t)=\frac{\pi \operatorname{cosec} \pi \alpha}{2^{\alpha} \Gamma(\alpha+1)} \int_{0}^{\infty} \xi^{2 \alpha} \frac{H(\xi) \chi_{v-\alpha, v}(u, \xi) \chi_{v-\alpha, v}(t, \xi)}{J_{v}^{2}(\xi)+Y_{v}^{2}(\xi)} d \xi
$$

The symmetrisation of the kernel can be done by substituting

$$
g(t) t^{\alpha-v}=t^{\frac{1}{2}} G(t) \text {. }
$$

\section{REFERENCES}

(1) H. Weser, Über die Besselschen Funktionen und ihre Anwendung an die Theorie der Elektrischen Ströme, J. f. Math., 75 (1873), 75.

(2) E. Beltrami, Sulla teoria della funzioni potenziali simmetriche, Rend. Acc. $d$. Sci. di Bologna (1881), 461.

(3) E. C. TIrchmarsh, Introduction to the Theory of Fourier Integrals (Oxford, 1937), p. 334. 115.

(4) I. W. Busbridge, Dual integral equations, Proc. London Math. Soc., 44 (1938),

(5) A. N. Gordon, Dual integral equations, J. London Math. Soc., 29 (1954), 360.

(6) I. N. SNeddon, The elementary solution of dual integral equations, Proc. Glasgow Math. Assoc., 4 (1960), 108.

(7) E. T. Copson, On certain dual integral equations, Proc. Glasgow Math. Assoc., 5 (1961), 21.

(8) B. Noble, On some dual integral equations, Quart. J. Math. (2), 6 (1955), 81. 60.

(9) C. J. Tranter, On some dual integral equations, Quart. J. Math. (2), 2 (1951),

(10) W. E. Williams, The solution of certain dual integral equations, Proc. Edinburgh Math. Soc., 12 (1961), 213.

(11) A. Erdelyi and I. N. SNeddon, Fractional integration and dual integral equations, Canadian J. Math., 14 (1962), 685.

(12) M. Lowengrub and I. N. SNeddon, The solution of a pair of dual integral equations, Proc. Glasgow Math. Assoc., 6 (1963), 14.

(13) W. E. Williams, Dual integral equations, Proc. Glasgow Math. Assoc. (to appear).

(14) J. C. Cooke and C. J. Tranter, Dual Fourier-Bessel series, Quart. J. Mech. Appl. Math., 12 (1959), 379.

(15) I. N. Sneddon and R. P. SRivastav, Dual Series Relations-I, Dual relations involving Fourier-Bessel series, Proc. Roy. Soc. Edinburgh, Series A, 66 (1962-63), $150-160$. 
(16) R. P. Srivastav, An axisymmetric mixed boundary value problem for a half-space with a cylindrical cavity, J. Math. Mech. 13 (1964), 385.

(17) C. J. Tranter, On some dual integral equations occurring in potential problems with axial symmetry, Quart. J. Mech. and Appl. Math., 3 (1950), 411.

(17a) C. J. Tranter, A further note on dual integral equations and an application to the diffraction of electromagnetic waves, Quart. J. Mech. and Appl. Math., 7 (1954), 318.

(18) J. C. Cooke, A solution of Tranter's dual integral equations problem, Quart. J. Mech. and Appl. Math., 9 (1956), 103.

(19) B. Noble, Certain dual integral equations, J. Math. Phys., 37 (1958), 128.

(20) G. N. WArson, A Treatise on the Theory of Bessel Functions (Cambridge University Press, 1944).

(21) A. ERDÉLYI, et al., Tables of Integral Transforms, Vol. 2 (McGraw Hill, 1954).

(22) E. C. Trtchmarsh, Eigenfunction Expansions associated with Second-order differential equations (Oxford, 1946).

Department of Mathematics

INDIAN INSTITUTE OF TECHNOLOGY

KaNPUR, (U.P.), INDIA 